\title{
Autoinducer-2 Facilitates Pseudomonas aeruginosa PAO1 Pathogenicity in Vitro and in Vivo
}

\begin{abstract}
Hongdong Li $i^{1,2,3,4}$, Xingyuan $\mathrm{Li}^{5}$, Chao Song ${ }^{1,2,3,4}$, Yunhui Zhang ${ }^{1,2,3,4}$, Zhengli Wang ${ }^{1,2,3,4}$, Zhenqiu Liu ${ }^{1,2,3,4}$, Hong Wei ${ }^{1,2,3,4}$ and Jialin $\mathrm{Yu}^{1,2,3,4,6 *}$

${ }^{1}$ Department of Neonatology, Children's Hospital of Chongqing Medical University, Chongqing, China, ${ }^{2}$ Ministry of Education Key Laboratory of Child Development and Disorders, Chongqing, China, ${ }^{3}$ China International Science and Technology Cooperation Base of Child Development and Critical Disorders, Chongqing, China, ${ }^{4}$ Chongqing Key Laboratory of Child Infection and Immunity, Chongqing, China, ${ }^{5}$ Department of Pharmacy, Chongqing Red Cross Hospital, Chongqing, China, ${ }^{6}$ Shenzhen University General Hospital, Shenzhen, China
\end{abstract}

Bacterial communication systems, such as quorum sensing (QS), have provided new insights of alternative approaches in antimicrobial treatment. We recently reported that one QS signal, named as autoinducer-2 (AI-2), can affect the behaviors of Pseudomonas aeruginosa PAO1 in a dose-dependent manner. In this study, we aimed to investigate the effects of $\mathrm{Al}-2$ on $P$. aeruginosa $\mathrm{PAO} 1$ biofilm formation and virulence factors production in vitro, and in vivo using a pulmonary infection mouse model. Exogenous Al-2 resulted in increased biofilms architecture, the number of viable cells, and the yield of pyocyanin and elastase virulence factors in wild type $P$. aeruginosa PAO1. However, no such effect was observed in $P$. aeruginosa las $R$ rhlR mutant strain. In vivo, the use of Al-2 significantly increased the mortality, lung bacterial count and histological lung damage of mice with acute $P$. aeruginosa PAO1 infection. Our data suggest that Al-2 promotes the formation of $P$. aeruginosa PAO1 biofilms and the production of virulence factors by interfering with $P$. aeruginosa QS systems, resulting in decreased host survival. Al-2 may be a therapeutic target for the clinical treatment of a co-infection of $P$. aeruginosa and Al-2 producing bacteria.

Keywords: Pseudomonas aeruginosa, autoinducer-2, biofilm, infection, virulence

\section{INTRODUCTION}

Pseudomonas aeruginosa is a versatile pathogenic bacterium, which is relevant to various acute and chronic infections in immunocompromised patients, such as those with cystic fibrosis, third-degree burns, and implanted medical devices (Mathee et al., 2008; Silby et al., 2011; Gellatly and Hancock, 2013). P. aeruginosa infection is difficult to eradicate due to its various virulence factors including pyocyanin, rhamnolipids, elastase, exotoxin A, phospholipase C (Ben Haj et al., 2011). In particular, the formation of biofilms leads to high morbidity and mortality among infected patients because biofilms are more resistant to chemical attacks and human immune responses than planktonic bacteria (Costerton et al., 1999; Ciofu et al., 2001; Singh et al., 2002).

When bacteria grow as biofilms, they are coordinately controlled by quorum sensing (QS), a process of cell-to-cell communication that modulates various behaviors in a population density-dependent manner (Miller and Bassler, 2001). As the number of QS signaling molecules accumulate to a threshold, the QS system could be activated by the identification of specific receptors (Miller and Bassler, 2001). The QS signaling molecules mainly contain two parts, 
oligopeptides are commonly secreted by Gram-positive bacteria, while $N$-acyl homoserine lactones are commonly secreted by Gram-negative bacteria (Miller and Bassler, 2001). Acyl homoserine lactone (AHL)-based QS systems in P. aeruginosa mainly involves the las and rhl systems, which encodes two specific signal molecules named $N$-(3-oxododecanoyl)L-homoserine lactone (3-oxo-C12-HSL) and $\mathrm{N}$-butanoyl-Lhomoserine lactone (C4-HSL), respectively (Pearson et al., 1994; Ochsner and Reiser, 1995; Miller and Bassler, 2001). In addition, researchers found that $P$. aeruginosa also employs the Pseudomonas quinolone signal (PQS) system, which can control the $r h l$ system activation (Diggle et al., 2003).

AI-2, named as a universal language among the communication between bacteria, could coordinate both intra- and interspecies communication (McNab et al., 2003). It is encoded by the LuxS gene, and is the primary QS molecule produced by many Gram-positive and Gram-negative bacteria (Cho et al., 2016). It could be produced by diverse genera (almost 50) and foster inter-species communication (Hardie and Heurlier, 2008). However, P. aeruginosa has no ability to produce AI-2, but it can sense the molecule and then affect its function (Li et al., 2015a). Previously, we and other researchers found that AI-2, as well as AI-2 producing Streptococcus mitis, could affect the behaviors of $P$. aeruginosa in vitro (Duan et al., 2003; Li et al., 2015a; Wang et al., 2016). Additionally, Roy et al. (2013) found that AI-2 analogs can reduce the virulence production of $P$. aeruginosa in vitro. Unfortunately, the effect of AI-2 on P. aeruginosa virulence in vivo remains unraveled. Since effects obtained in in vitro model systems cannot always be repeated under in vivo conditions (Brackman et al., 2011), it was unclear whether AI-2 could also influence $P$. aeruginosa infections in vivo. Furthermore, there is a lack of direct evidence to illustrate the mechanisms of AI-2.

In the present study, we aimed to investigate the effects of AI-2 on $P$. aeruginosa biofilm formation and virulence factors production in vitro, as well as in vivo using a pulmonary infection mouse model, and to explore the roles of AI-2 in P. aeruginosa infections. This work may provide important insights to combat $P$. aeruginosa infections.

\section{MATERIALS AND METHODS}

\section{In Vitro Experiments}

\section{Bacterial Strains and Culture Conditions}

Pseudomonas aeruginosa PAO1 (ATCC 27853) and its double mutant $P$. aeruginosa lasR rhlR (Wu et al., 2001; Feng et al., 2016) were as gifts provided by Professor Li Shen (Institute of Molecular Cell and Biology, New Orleans, LA, United States) and Professor Zhijun Song (Faculty of Health and Medical Sciences, University of Copenhagen, Copenhagen, Denmark), respectively. Both bacteria were incubated at $37^{\circ} \mathrm{C}$ with shaking $(200 \mathrm{rpm})$. Chemically synthesized AI-2 precursor DPD $[(S)$ 4, 5-dihydroxy-2, 3-pentanedione] was obtained from Omm Scientific company (Dallas, TX, United States). The AI-2 used in this study was chosen as a biological relevant concentration, because $10 \mathrm{nM}$ AI-2 resulted in the greatest response in virulence factor production and biofilm formation (Li et al., 2015a). Furthermore, AI-2 producing S. mitis and Klebsiella pneumoniae were the most frequent microbes in the biofilms on the surface of neonatal endotracheal tubes extubated from mechanically ventilated newborns, and the AI-2 concentration secreted by these AI-2 producing bacteria in the biofilms on the surface of neonatal endotracheal tubes was about 10-50 nM (Li et al., 2015b; Wang et al., 2016; Pan et al., 2017).

\section{Biofilm Formation Assays}

The biofilm formation assay was performed under a static environment in 96-well polystyrene microtiter plates as previously described (Sarkar et al., 2014). In brief, an optical density at $600 \mathrm{~nm}\left(\mathrm{OD}_{600}\right)$ of 0.05 diluted cultures with 10 nM AI-2 were added to 96-well microtiter plates (Costar, United States), and 8 replicates were added in each group. After $24 \mathrm{~h}$ at $37^{\circ} \mathrm{C}$ without shaking, the plates were washed by phosphate-buffered saline (PBS) at least three times. Then, the plates were dried and stained with $0.1 \%$ crystal violet. The bacteria-bound crystal violet was dissolved in $200 \mu \mathrm{L} 95 \%$ ethanol, and the absorbance at $570 \mathrm{~nm}$ was determined. All experiments were performed three times independently $(n=24)$.

\section{Confocal Laser Scanning Microscopy (CLSM) and Viability Counts}

Pseudomonas aeruginosa PAO1 and P. aeruginosa lasR rhlR mutant cells were inoculated in LB broth at a standard concentration $(\mathrm{A} 600=0.05)$, then the dilutions were added to glass coverslips (Costar, United States) which were laid in a 24well plate ( 3 replicates were added in each group), followed by growth for $48 \mathrm{~h}$ without shaking at $37^{\circ} \mathrm{C}$. Coverslips were then washed three times with PBS and stained with SYTO9/propidium iodide according to the manufacturer's instructions of the L13152 LIVE/DEAD BacLight bacterial viability kit (Invitrogen Molecular Probes, United States). Coverslips were visualized by a Nikon A1R laser confocal microscope (Nikon, Tokyo, Japan). Live bacteria were stained green (excitation $488 \mathrm{~nm}$, emission $515 / 30 \mathrm{~nm}$ ) while dead bacteria were stained red (excitation $568 \mathrm{~nm}$, emission 600/50 nm). In parallel experiments, samples were also prepared for viability counts at 24 and $48 \mathrm{~h}$, respectively.

\section{Virulence Factor Assays}

For the pyocyanin assay, $3 \mathrm{~mL}$ supernatant from a $24 \mathrm{~h}$ culture in pyocyanin production broth $(\mathrm{PPB} ; 2 \%$ proteose peptone [Oxoid, UK], $1 \% \mathrm{~K}_{2} \mathrm{SO}_{4}, 0.3 \% \mathrm{MgCl}_{2} \cdot 6 \mathrm{H}_{2} \mathrm{O}$ ) was extracted by chloroform, and further re-extracted with $0.2 \mathrm{M} \mathrm{HCl}$, a red layer was obtained. The optical density was determined at $520 \mathrm{~nm}$ (Kong et al., 2005). Elastase activity in the supernatant was processed using the elastin-Congo red (ECR) assay as previously described (Yang et al., 2012). Briefly, filtered supernatant from a $6 \mathrm{~h}$ culture in peptone tryptic soy broth (PTSB; 5\% peptone, $0.1 \%$ tryptic soy broth) was added to a new tube containing $10 \mathrm{mg}$ of ECR (Sigma, United States), $900 \mu \mathrm{L} 10 \mathrm{mM}$ Tris $\mathrm{HCl}$ ( $\mathrm{pH} 7.5$ ), and $1 \mathrm{mM} \mathrm{CaCl}_{2}$. The tubes were incubated for $4 \mathrm{~h}$ at $37^{\circ} \mathrm{C}$ with agitation. At last, unreacted substrate was removed by centrifugation. The optical density was determined at $495 \mathrm{~nm}$. 
Five replicates were in each group and all experiments were performed three times independently.

\section{In Vivo Experiments Animals}

All experiments were performed in accordance with relevant guidelines and regulations, and the animal experimental protocol was approved by the Animal Care and Use Committee at the Chongqing Medical University. Male C57BL/6 mice aged 6-8 weeks were purchased from Chongqing Medical University (Chongqing, China). All mice were housed in individually filtered cages and received unlimited sterile food and water in the Laboratory Animal Center at the Children's Hospital of Chongqing Medical University.

\section{Mouse Model of $P$. aeruginosa Acute Lung Infection}

An experimental model of acute lung infection was established following the method of Song et al. with some modifications (Song et al., 2014). The concentration of $P$. aeruginosa cultures were adjusted to $0.25\left(\mathrm{~A}_{600}\right)$. Inoculated bacterial counts were confirmed by plating the suspensions on LB plates (Sangon, Shanghai, China). Mice were infected with an intratracheal instillation of $0.05 \mathrm{ml}$ of bacterial suspension $\left(1 \times 10^{7}\right.$ colonyforming units/mouse) under anesthesia with pentobarbital sodium. As control, intratracheal instillation was performed with $0.05 \mathrm{ml} \mathrm{PBS}$ or $10 \mathrm{nM} \mathrm{AI-2}$ in the same manner. Thus, there were four groups used in the study: (i) infection control group: infection was given with $\mathrm{PAO} 1$ only ( $\mathrm{P}$ group); (ii) AI-2 treated group: infection was given with PAO1 and AI-2 (P+AI-2 group); (iii) AI-2 control group: treatment was given with AI-2 only (AI-2 group); (iv) PBS control group: treatment was given with PBS only (PBS group). For survival studies, 12 mice in each group were observed once daily for 7 days post infection. Other mice (a total of 60 mice with 5 mice in each group for each experiment) were sacrificed by cervical dislocation after $24 \mathrm{~h}$ of the infection, and the lungs and bronchoalveolar lavage fluid (BALF) were collected for further analysis."(see below)."

\section{Bacteriological and Histopathological Examination}

For bacteriological analysis, the lungs were suspended in $1 \mathrm{ml}$ of cold sterile PBS and homogenized using a homogenizer (Changzheng Co., Chongqing, China) at $4^{\circ} \mathrm{C}$. The specimen was serially diluted and plated on $P$. aeruginosa selective agar to measure the CFU per lung, followed by incubation at $37^{\circ} \mathrm{C}$ for 16-20 h. For histopathological analysis, lung tissues were fixed in $10 \%$ neutral buffered formalin for $48 \mathrm{~h}$, and the paraffin-embedded sections, cut into $5 \mu \mathrm{m}$ sections, were stained with hematoxylin-eosin (H\&E; Sigma). Light microscopy (Nikon eclipse 55i, Japan) was used to obtain the images.

\section{Analysis of BALF}

Mice were anesthetized with pentobarbital sodium at $24 \mathrm{~h}$ after $P$. aeruginosa infection, an appropriate plastic intravenous catheter was inserted into the trachea, BALF was collected by three times washes with ice-cold PBS, and total cell numbers in BALF were measured. Cell-free BALF supernatants were collected by centrifugation (3000 $\mathrm{g}$ for $5 \mathrm{~min}$ at $4^{\circ} \mathrm{C}$ ) and stored at $-80^{\circ} \mathrm{C}$ prior to cytokine profile characterization. The pro-inflammatory cytokines tumor necrosis factor alpha (TNF- $\alpha)$, IL-6 and the anti-inflammatory cytokine IL-10 concentration in the supernatant fluid were determined by using mouse cytokine enzyme-linked immunosorbent assay kits (Sizhengbai, Beijing, China) according to the manufacturer's instructions.

\section{Statistical Analysis}

All data from the study are expressed as means \pm SD. Analysis of variance (ANOVA) was used to determine the level of significant differences between all groups and Tukey's honest significant difference (HSD) for pairwise comparison. Survival analysis was performed using the log-rank (MantelCox) test. $P<0.05$ was considered to be statistically significant.

\section{RESULTS}

\section{In Vitro Experiments}

Al-2 Increased $P$. aeruginosa PAO1 Biofilm Formation First, we monitored wild type $P$. aeruginosa PAO1 biofim formation with and without $10 \mathrm{nM}$ AI-2. Crystal violet assay revealed that $\mathrm{AI}-2$ added culture ( $\mathrm{P}+\mathrm{AI}-2$ group) resulted in a significant increase in the total biomass by $61.4 \%$ as compared to PAO1 control group (P group) (Figure 1A). Notably, consistent with biomass results, Two-dimensional (2D) imaging revealed a more dense biofilm architecture under CLSM in the P+AI-2 group, and more viable cells were visualized (Figure 1B). In addition, the number of viable bacteria in the $\mathrm{P}+\mathrm{AI}-2$ group was significantly higher than that in the $\mathrm{P}$ group (Table 1), the amount of bacteria released from the biofilms at $24 \mathrm{~h}$ in the $\mathrm{P}+\mathrm{AI}-2$ group increased by $80.6 \%$ as compared to $\mathrm{P}$ group $\left[(5.6 \pm 0.5) \times 10^{7} \mathrm{CFU} / \mathrm{cm}^{2}\right.$ vs. $\left.(3.1 \pm 0.4) \times 10^{7} \mathrm{CFU} / \mathrm{cm}^{2}, F(3,36)=23.25, P=0.03\right]$. In addition, we used the same method to assess the effect of AI-2 on $P$. aeruginosa las $R$ rhlR mutant strain. The results showed that biofilm formation of the mutant strain was impaired when compared to the wild type PAO1. Furthermore, the addition of AI-2 to $P$. aeruginosa las $R$ rhlR mutant cultures $(\Delta \mathrm{P}+\mathrm{AI}-$ 2 group) resulted in no significant changes as compared to $P$. aeruginosa las $R$ rhlR mutant group ( $\triangle \mathrm{P}$ group) (Figure 1 and Table 1).

\section{Al-2 Increased $P$. aeruginosa PAO1 Virulence Factors Production}

Pyocyanin and elastase, controlled by the P. aeruginosa QS system, are two very important virulence factors (Ben Haj et al., 2011). Whether AI-2 could promote the production of these two virulence factors was determined. As shown in Figure 2, the activity of both pyocyanin and elastase were significantly increased in the P+AI-2 group $[F(3,57)=99.03, P=0.004$ and $F(3,57)=162.33, P=0.001$, respectively] while there was no such effect in $\Delta \mathrm{P}+\mathrm{AI}-2$ group. 
A

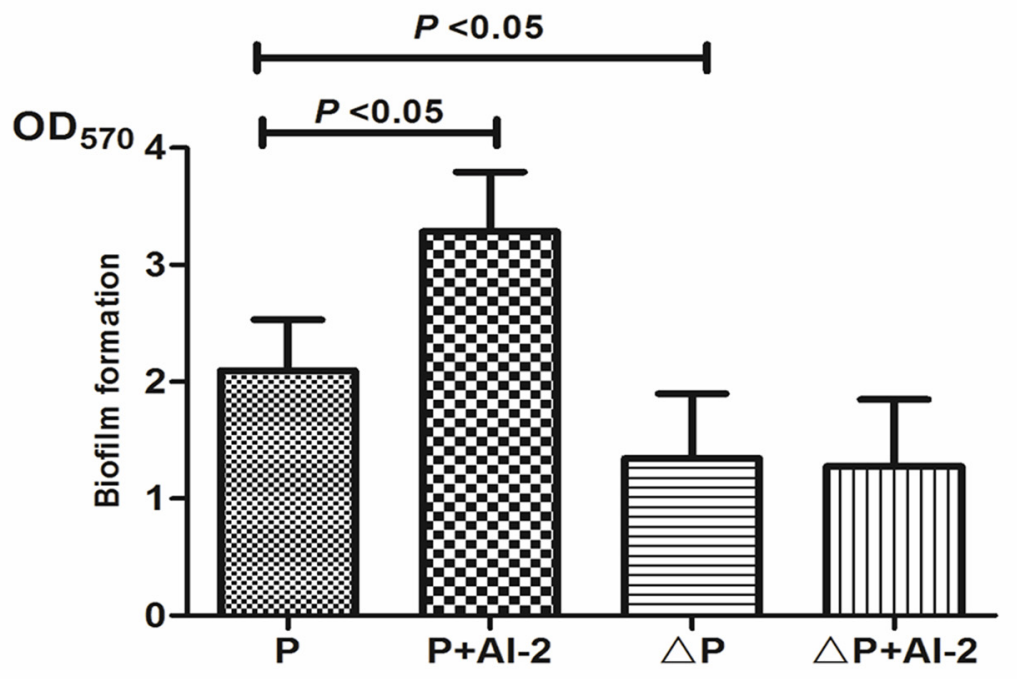

B
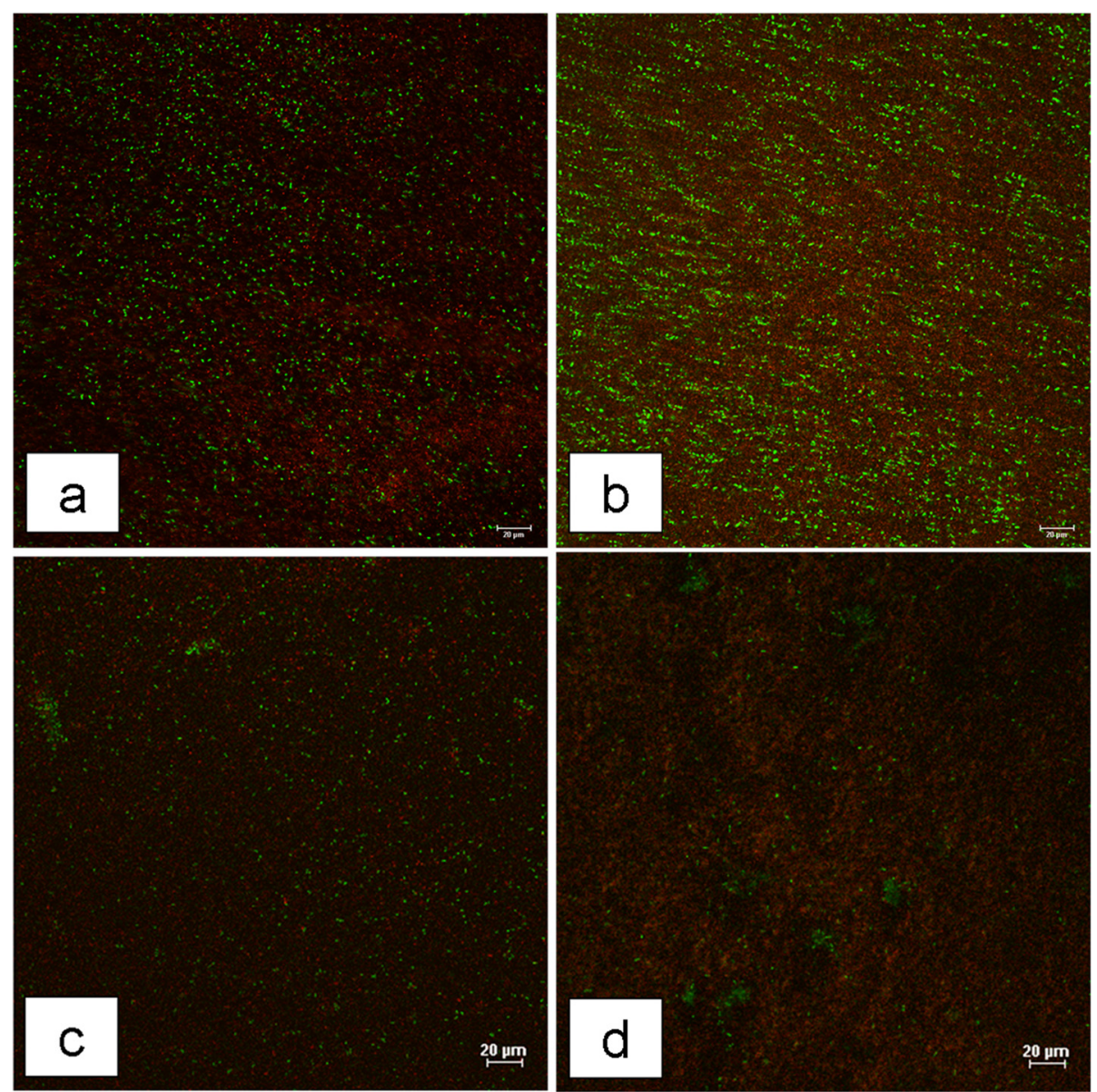

FIGURE 1 | Effects of $\mathrm{Al}-2$ on $\mathrm{P}$. aeruginosa $\mathrm{PAO} 1$ biofilm formation. $\mathrm{P}, \mathrm{PAO} 1$ group; $\mathrm{P}+\mathrm{Al}-2, \mathrm{PAO} 1+\mathrm{Al}-2$ group; $\Delta \mathrm{P}, \mathrm{P}$. aeruginosa lasR rhlR mutant group; $\Delta \mathrm{P}+\mathrm{Al}-2$, $P$. aeruginosa las $R$ rhIR mutant+Al-2 group. (A) Biofilm formation was assessed by crystal violet. Error bars represent $S D$ and all experiments were performed in three times independently $(n=24)$; (B) Representative confocal laser scanning micrographs of biofilms in four groups. Compared to the PAO1 control group (a), a dense and compact biofilm and more live bacteria were observed under CLSM in P+Al-2 group (b) and a loosend biofilm structure and fewer viable cells were visualized in the $\Delta \mathrm{P}+\mathrm{Al}-2$ group (c) and $\Delta \mathrm{P}$ group (d). Red, non-viable cells; green, viable cells. scale bars $=20 \mu \mathrm{m}$. 
TABLE 1 | Viable bacterial counts recovered from biofilms formed on coverslips.

\begin{tabular}{|c|c|c|}
\hline \multirow[t]{2}{*}{ Groups } & \multicolumn{2}{|c|}{ CFU $/ \mathrm{cm}^{2}$ biofilm } \\
\hline & $24 \mathrm{~h}$ & $48 \mathrm{~h}$ \\
\hline$P$ & $(3.1 \pm 0.4) \times 10^{7}$ & $(1.2 \pm 0.3) \times 10^{8}$ \\
\hline $\mathrm{P}+\mathrm{Al}-2$ & $(5.6 \pm 0.5) \times 10^{7 *}$ & $(3.2 \pm 0.6) \times 10^{8} \Delta$ \\
\hline$\Delta \mathrm{P}$ & $(7.5 \pm 0.38) \times 10^{6 *}$ & $(5.8 \pm 0.5) \times 10^{7} \Delta$ \\
\hline$\Delta \mathrm{P}+\mathrm{Al}-2$ & $(9.2 \pm 0.4) \times 10^{6}$ & $(6.6 \pm 0.43) \times 10^{7}$ \\
\hline
\end{tabular}

\section{In Vivo Experiments}

\section{Al-2 Increased Mortality Rates in Mouse Models of Lung Infection}

To evaluate the effects of AI-2 on mouse models in vivo, we first determined the mortality rates of the animals which were coinfected with AI-2 or not. The mortality rate was $0(0 / 12)$ in the PBS or AI-2 group (Figure 3A), indicating that AI-2 itself has no effect on animals. The spontaneous mortality rate was $50 \%(6 / 12)$ in the PAO1 control group (P). However, when the mice were co-infected with $P$. aeruginosa $\mathrm{PAO} 1$ and $\mathrm{AI}-2$, the mortality rate was significantly increased to $66.7 \%(8 / 12)(P<0.05)$.

\section{Al-2 Increased $P$. aeruginosa Local Infection in the Lungs}

We further collected the lungs which were infected $24 \mathrm{~h}$ post infection for bacteriological analysis. There was no $P$. aeruginosa colonizing in the lungs in AI-2 group and PBS group. However, the bacterial count in the lungs of $\mathrm{P}+\mathrm{AI}-2$ group $(5.2 \pm 0.15 \mathrm{Log}$ $\mathrm{CFU} / \mathrm{g})$ was significantly more than that in $\mathrm{P}$ group $(4.4 \pm 0.1$ $\log \mathrm{CFU} / \mathrm{g})[\mathrm{F}(3,17)=102.5, P=0.004]$ (Figure 3B).

\section{Al-2 Aggravated $P$. aeruginosa -Induced Lung Injury and Inflammation}

To evaluate the pro-inflammatory effects of AI-2, lung tissues were harvested post infection. The $\mathrm{P}+\mathrm{AI}-2$ group exhibited a higher bacterial loads and more serious pathological changes. In $\mathrm{P}+\mathrm{AI}-2$ group mice, more leukocytes were found to infiltrate into the peribronchiolar and perivascular connective tissues, and the neutrophil cells were the primary infiltrating cell types (Figure 4). Moreover, to examine whether AI-2 could aggravate the inflammatory cell infiltration into the lungs following PAO1 infection, the total cells present in the BALF were counted. As shown in Figure $\mathbf{5 A}$, the median total number of BALF cells in $\mathrm{P}+\mathrm{AI}-2$ group was $4.75 \times 10^{6}$ cells/mouse, as compared to $3.5 \times 10^{6}$ cells/mouse in $\mathrm{P}$ group $[F(3,17)=153.25, P=0.002]$. To further assess the effects of AI- 2 on BALF cytokine profile changes, ELISA-based assays were used. The levels of cytokines (TNF- $\alpha$, IL-6) in the BALF were significantly higher in the P+AI2 group than those in the $\mathrm{P}$ group $[F(3,17)=91.03, P=0.005$ and $F(3,17)=162.33, P=0.001$, respectively] (Figures 5B,D). IL-10 levels tended to be higher in the $\mathrm{P}+\mathrm{AI}-2$ group than in the $\mathrm{P}$ group, although the increase was not statistically significant

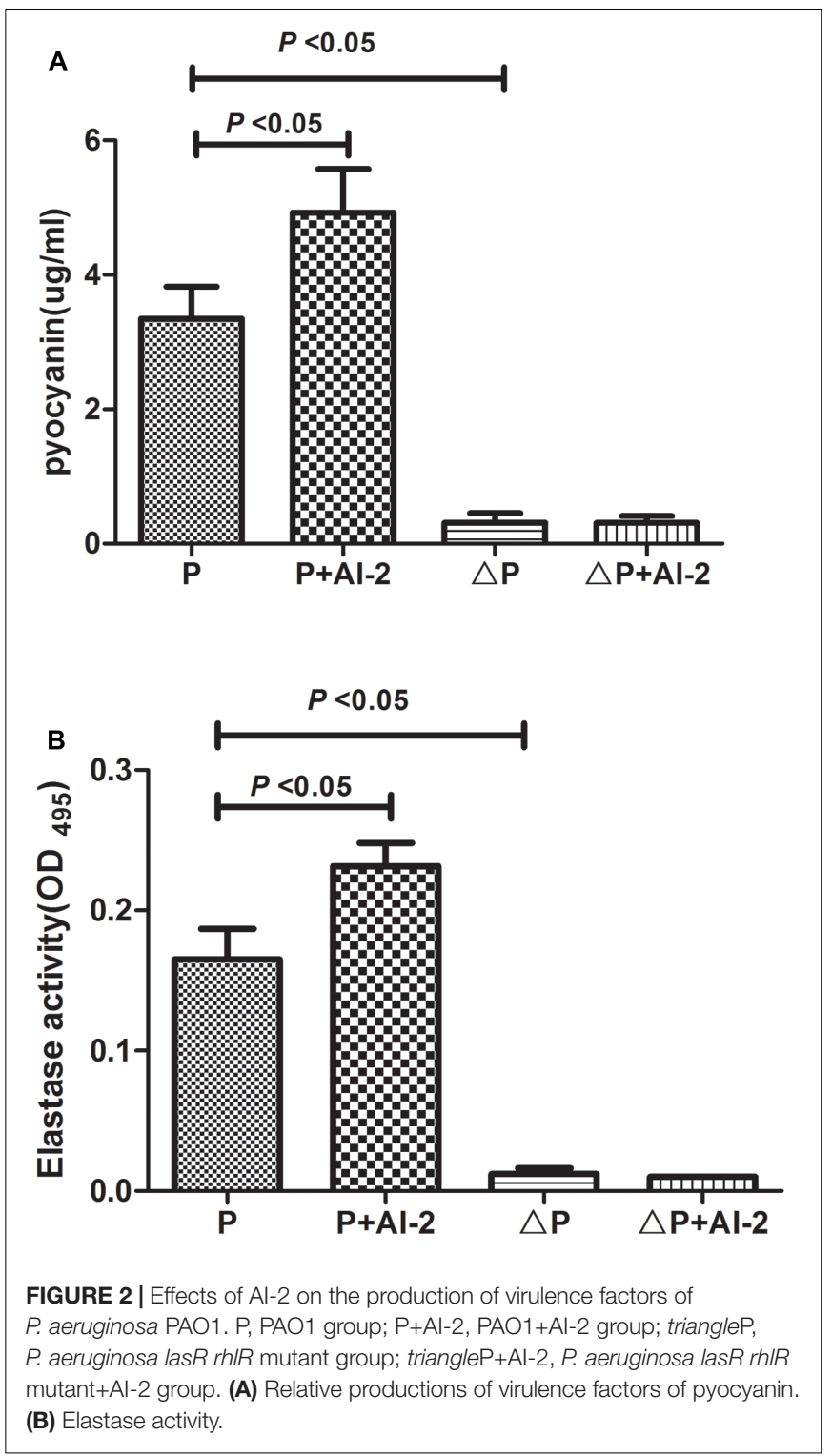

(Figure 5C). Therefore, these results suggest that AI-2 could aggravate lung injury and inflammation by increasing bacterial counts.

\section{DISCUSSION}

In this study, both in vitro and in vivo results demonstrated that AI-2 could influence $P$. aeruginosa PAO1 biofilm formation and virulence factor production by interfering with $P$. aeruginosa $\mathrm{QS}$ systems, leading to an increased lung infection and mortality rate. $P$. aeruginosa is a common bacterium that can cause various serious infections in human body (Brennan and Geddes, 2002). Recently, we found it is also one of the most prevalent bacteria on endotracheal tubes (ETT) samples of neonates who were under mechanical ventilation (Li et al., 2015b). One interesting phenomenon is that AI-2 producers Klebsiella spp. 

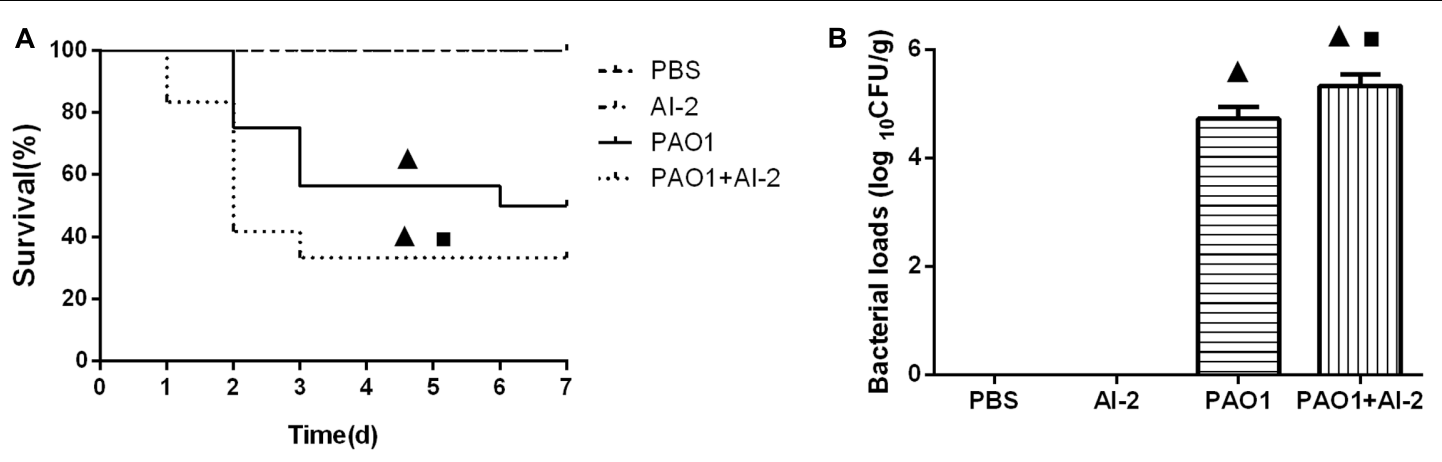

FIGURE 3 | Analysis of survival data and bacterial loads after infection. The animals were treated with PBS, Al-2, P. aeruginosa PAO1 (P) and Al-2+P. aeruginosa PAO1 $(\mathrm{P}+\mathrm{Al}-2)$, respectively. Triangles denote a statistically significant difference from the PAO1 group $(P<0.05)$, asterisks denote a statistically significant difference from the PBS group ( $P<0.05$ ). (A) Survival was monitored for 7 days ( $n=12$ mice per group); (B) Bacterial loads in the lungs after infection for 24 h.
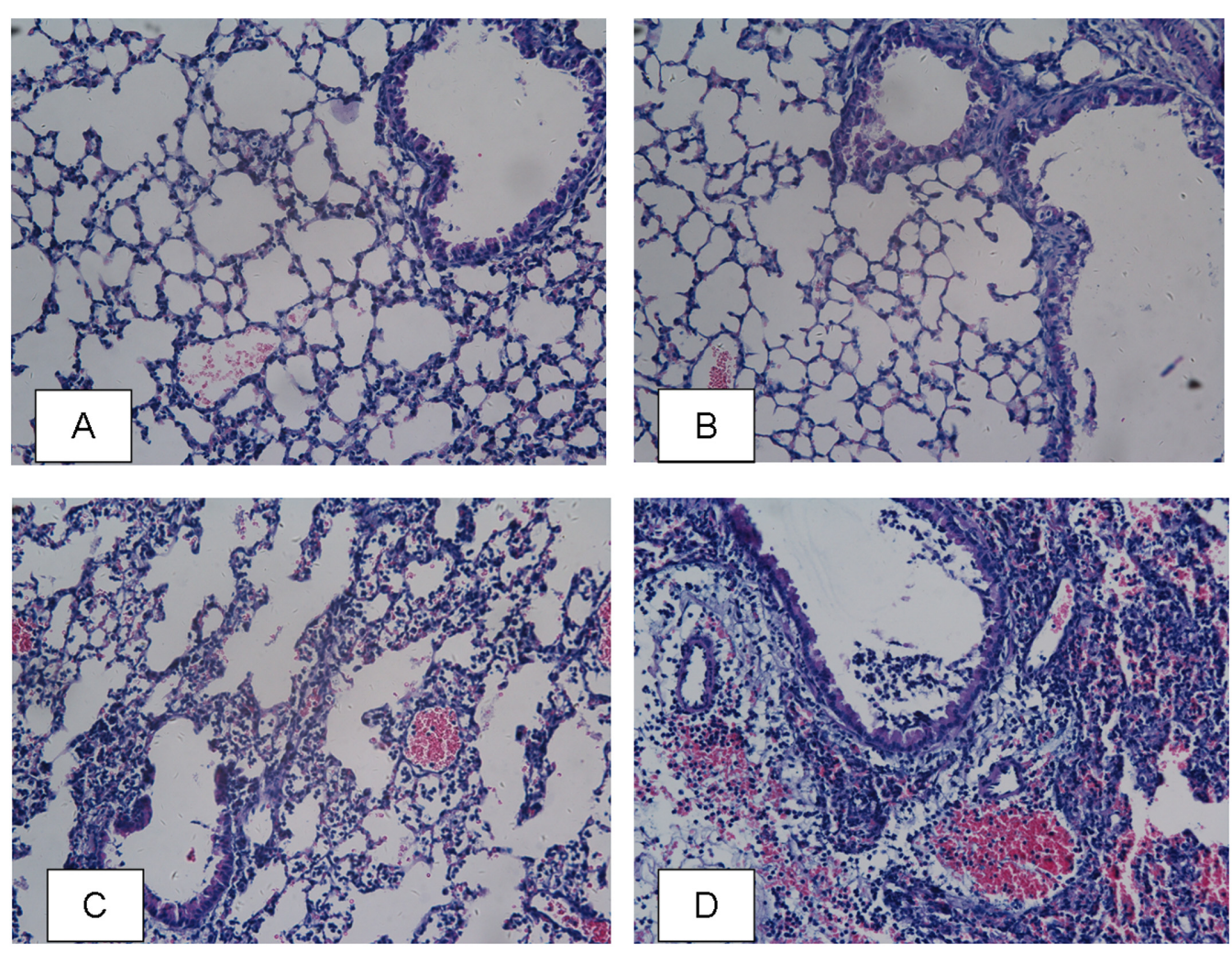

FIGURE 4 | Lung histological examination after infection. Sections of lungs stained with hematoxylin-eosin at $24 \mathrm{~h}$ post infection are shown (magnifications, $\times 400$ ). (A) PBS group; (B) Al-2 group; (C) P. aeruginosa PAO1 group; (D) P. aeruginosa PAO1+Al-2 group.

and Streptococcus spp. often coexist with $P$. aeruginosa on the same ETT samples (Li et al., 2015b). Biofilms harbor a complex microbiological community, which means a specific environment on the surface of neonatal ETTs. As AI-2 is a universal signal for intra- and interspecies communication, AI-2 signaling pathway is a potential target to inhibit biofilms infections (Jang et al., 2013; Roy et al., 2013).

The role of AI-2 as a general bacterial signaling molecule affecting biofilm formation in vitro has been widely studied. AI-2 inhibits biofilm formation in Bacillus cereus (Auger et al., 2006), Candida albicans (Bachtiar et al., 2014), and Eikenella corrodens (Azakami et al., 2006), and it promotes biofilm formation in Escherichia coli (Gonzalez Barrios et al., 2006), Streptococcus mutans (Yoshida et al., 2005), and multispecies biofilms between the two oral bacteria Streptococcus gordonii and Porphyromonas gingivalis (McNab et al., 2003). However, to the best of our knowledge, the addition of exogenous AI-2 to bacterial acute pneumonia is seldom investigated, and this is the first study 


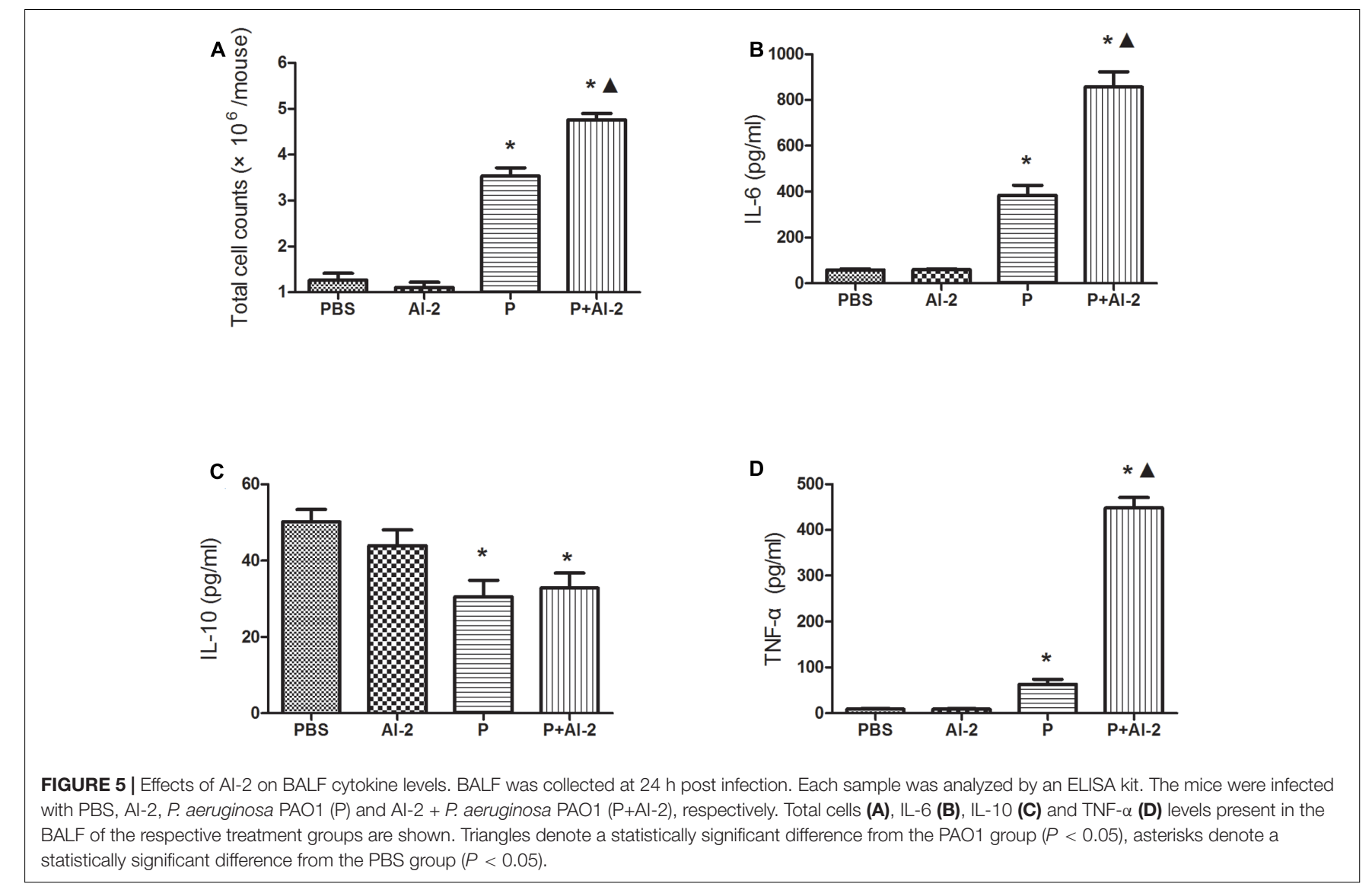

aimed at revealing the AI-2 effects on P. aeruginosa infections in vivo.

Based on the positive interaction observed between AI-2 and $P$. aeruginosa in in vitro studies, we further established a pulmonary infection mouse model to investigate the effects of AI-2 on $P$. aeruginosa infections in vivo. Lower rates of survival and more serious infections were observed in the $\mathrm{P}+\mathrm{AI}-2$ group than in the $\mathrm{P}$ group as a result of higher bacterial loads, more serious pathological changes, and more inflammatory cells infiltrating in the BALF. Similarly, Duan et al. (2003) reported that AI-2 producing bacteria could aggravate the lung injury when co-infected with $P$. aeruginosa. This phenomenon may be attributed to the higher secretion of elastase and pyocyanin, which was regulated by QS genes and virulence factor genes. We previously found that AI-2 could enhance the expression of the $P$. aeruginosa PAO1 QS genes and virulence factor genes, including lasI, lasR, rhlI, rhlR, lasB, las A, and $p h z H$ (Li et al., 2015a). Pyocyanin in the lungs of mice has been shown to cause a number of pathological changes including airway fibrosis, goblet cell hyperplasia and metaplasia, and destruction of the alveolar airspaces (Caldwell et al., 2009). Furthermore, Yilmaz et al. (2015) found that pyocyanin could contribute to the virulence of Porphyromonas gingivalis and disease severity. Besides, elastase could break down hemoglobin and lead to pulmonary hemorrhage (Cosgrove et al., 2011). In this study, the concentration of IL-10 in the BALF had no significant difference between $\mathrm{P}$ group and $\mathrm{P}+\mathrm{AI}-2$ group $(P>0.05)$, but both groups showed significantly higher concentration than in PBS control group $(P<0.05)$. IL-10 is an anti-inflammatory cytokine and a key immunoregulator during infection. High pathogen loads drive immoderate Th1 responses (Anderson et al., 2005), and in turn, promotes the development of self-limiting inflammation and adaptive immune responses. IL-10-producing T cells could dampen the Th1 response (Belkaid et al., 2001; Anderson et al., 2007). These events form a positive feedback loop whereby IL-10 further inhibits antimicrobial immune responses, allowing inevitably serious infections to develop (Couper et al., 2008).

QS is known to play a key role in biofilm formation and virulence production (Miller and Bassler, 2001). Thus, it can be a suitable target for antimicrobial therapy. It is noteworthy that the $P$. aeruginosa lasR $r h l R$ mutant has weaker biofilm formation and reduced virulence factor production as compared with PAO1 (Figures 1, 2). Meanwhile, P. aeruginosa QS mutations are very common in isolates from cystic fibrosis patients with chronic infection (Jiricny et al., 2014). Currently, chemicals that interfere with QS systems are being studied. Brackman et al. (2011) found that quorum-sensing inhibitors (QSI) increased the susceptibility of bacterial biofilms to antibiotics both in vitro and in vivo. Christensen et al. found that the QSIs furanone C-30, ajoene or horseradish juice extract could have a synergistic antimicrobial efficacy when used in combination with tobramycin (Christensen et al., 2012). Furthermore, we have demonstrated that D-ribose 
(an AI-2 analog) could reverse the effects produced by two common pathogens on ETT, S. mitis and P. aeruginosa (Wang et al., 2016). However, the results from our study seemed promising, and it may be of significance to determine whether these effects can be obtained for other common pathogens on ETT (such as Klebsiella pneumoniae). On the other hand, we would further explore if AI-2 could enhance the biofilm formation of $P$. aeruginosa in in vivo models and the cytotoxicity of AI-2 as higher concentrations might be toxic. Hoffmann et al. (Hoffmann et al., 2005) found that biofilms were established in a mouse model of chronic $P$. aeruginosa lung infection mimicking cystic fibrosis. In future studies, it will therefore be important to clarify these mechanisms.

\section{CONCLUSION}

This study demonstrated that AI-2 increased $P$. aeruginosa PAO1 pathogenicity both in vitro and in vivo, and AI-2 increased the mortality rate and aggravated the lung infection in a mouse model of acute $P$. aeruginosa pneumonia by secreting more virulence factors. Our results support the significance

\section{REFERENCES}

Anderson, C. F., Mendez, S., and Sacks, D. L. (2005). Nonhealing infection despite Th1 polarization produced by a strain of Leishmania major in C57BL/6 mice. J. Immunol. 174, 2934-2941. doi: 10.4049/jimmunol.174.5.2934

Anderson, C. F., Oukka, M., Kuchroo, V. J., and Sacks, D. (2007). CD4(+)CD25(-)Foxp3(-) Th1 cells are the source of IL-10-mediated immune suppression in chronic cutaneous leishmaniasis. J. Exp. Med. 204, 285-297. doi: 10.1084/jem.20061886

Auger, S., Krin, E., Aymerich, S., and Gohar, M. (2006). Autoinducer 2 affects biofilm formation by Bacillus cereus. Appl. Environ. Microbiol. 72, 937-941. doi: 10.1128/aem.72.1.937-941.2006

Azakami, H., Teramura, I., Matsunaga, T., Akimichi, H., Noiri, Y., Ebisu, S., et al. (2006). Characterization of autoinducer 2 signal in Eikenella corrodens and its role in biofilm formation. J. Biosci. Bioeng. 102, 110-117. doi: 10.1263/jbb. 102.110

Bachtiar, E. W., Bachtiar, B. M., Jarosz, L. M., Amir, L. R., Sunarto, H., Ganin, H., et al. (2014). AI-2 of Aggregatibacter actinomycetemcomitans inhibits Candida albicans biofilm formation. Front. Cell Infect. Microbiol. 4:94. doi: 10.3389/ fcimb.2014.00094

Belkaid, Y., Hoffmann, K. F., Mendez, S., Kamhawi, S., Udey, M. C., Wynn, T. A., et al. (2001). The role of interleukin (IL)-10 in the persistence of Leishmania major in the skin after healing and the therapeutic potential of anti-IL-10 receptor antibody for sterile cure. J. Exp. Med. 194, 1497-1506. doi: 10.1084/ jem.194.10.1497

Ben Haj, Khalifa, A., Moissenet, D., Vu Thien, H., and Khedher, M. (2011). Virulence factors in Pseudomonas aeruginosa: mechanisms and modes of regulation. Ann. Biol. Clin. 69, 393-403. doi: 10.1684/abc.2011.0589

Brackman, G., Cos, P., Maes, L., Nelis, H. J., and Coenye, T. (2011). Quorum sensing inhibitors increase the susceptibility of bacterial biofilms to antibiotics in vitro and in vivo. Antimicrob. Agents Chemother. 55, 2655-2661. doi: 10.1128/aac.00045-11

Brennan, A. L., and Geddes, D. M. (2002). Cystic fibrosis. Curr. Opin. Infect. Dis. 15, 175-182. doi: 10.1097/00001432-200204000-00013

Caldwell, C. C., Chen, Y., Goetzmann, H. S., Hao, Y., Borchers, M. T., Hassett, D. J., et al. (2009). Pseudomonas aeruginosa exotoxin pyocyanin causes cystic fibrosis airway pathogenesis. Am. J. Pathol. 175, 2473-2488. doi: 10.2353/ajpath.2009. 090166

Cho, Y. J., Song, H. Y., Ben Amara, H., Choi, B. K., Eunju, R., Cho, Y. A., et al. (2016). In vivo inhibition of Porphyromonas gingivalis growth and prevention of AI-2 in the regulation of $P$. aeruginosa PAO1. AI-2 may provide novel means of combating clinical co-infection of $P$. aeruginosa and AI-2 producer bacteria by interfering with bacterial signaling.

\section{AUTHOR CONTRIBUTIONS}

HL and JY conceived and designed this study. HL, CS, ZW, and YZ performed the experiments. HL, XL, ZL, and HW analyzed the data and wrote the paper. HL, ZL, and HW revised the manuscripts.

\section{FUNDING}

This study was supported by the National Natural Science Foundation of China (Nos. 81370744, 81571483), Doctoral Degree Funding from Chinese Ministry of Education (No. 20135503110009), State key clinic discipline project (No. 2011873), Clinical Research Foundation of Children's Hospital of Chongqing Medical University (No. (2014) 254-lcyj2014-11).

of periodontitis with quorum-sensing inhibitors. J. Periodontol. 87, 1075-1082. doi: 10.1902/jop.2016.160070

Christensen, L. D., van Gennip, M., Jakobsen, T. H., Alhede, M., Hougen, H. P., Hoiby, N., et al. (2012). Synergistic antibacterial efficacy of early combination treatment with tobramycin and quorum-sensing inhibitors against Pseudomonas aeruginosa in an intraperitoneal foreign-body infection mouse model. J. Antimicrob. Chemother. 67, 1198-1206. doi: 10.1093/jac/ dks002

Ciofu, O., Fussing, V., Bagge, N., Koch, C., and Hoiby, N. (2001). Characterization of paired mucoid/non-mucoid Pseudomonas aeruginosa isolates from Danish cystic fibrosis patients: antibiotic resistance, beta-lactamase activity and RiboPrinting. J. Antimicrob. Chemother. 48, 391-396. doi: 10.1093/jac/48.3.391

Cosgrove, S., Chotirmall, S. H., Greene, C. M., and McElvaney, N. G. (2011). Pulmonary proteases in the cystic fibrosis lung induce interleukin 8 expression from bronchial epithelial cells via a heme/meprin/epidermal growth factor receptor/Toll-like receptor pathway. J. Biol. Chem. 286, 7692-7704. doi: 10.1074/jbc.M110.183863

Costerton, J. W., Stewart, P. S., and Greenberg, E. P. (1999). Bacterial biofilms: a common cause of persistent infections. Science 284, 1318-1322. doi: 10.1126/ science.284.5418.1318

Couper, K. N., Blount, D. G., and Riley, E. M. (2008). IL-10: the master regulator of immunity to infection. J. Immunol. 180, 5771-5777. doi: 10.4049/jimmunol. 180.9.5771

Diggle, S. P., Winzer, K., Chhabra, S. R., Worrall, K. E., Camara, M., and Williams, P. (2003). The Pseudomonas aeruginosa quinolone signal molecule overcomes the cell density-dependency of the quorum sensing hierarchy, regulates rhl-dependent genes at the onset of stationary phase and can be produced in the absence of LasR. Mol. Microbiol. 50, 29-43. doi: 10.1046/j.13652958.2003.03672.x

Duan, K., Dammel, C., Stein, J., Rabin, H., and Surette, M. G. (2003). Modulation of Pseudomonas aeruginosa gene expression by host microflora through interspecies communication. Mol. Microbiol. 50, 1477-1491. doi: 10.1046/j. 1365-2958.2003.03803.x

Feng, L., Xiang, Q., Ai, Q., Wang, Z., Zhang, Y., and Lu, Q. (2016). Effects of quorum sensing systems on regulatory T cells in catheter-related Pseudomonas aeruginosa biofilm infection rat models. Mediators Inflamm. 2016:4012912. doi: 10.1155/2016/4012912

Gellatly, S. L., and Hancock, R. E. (2013). Pseudomonas aeruginosa: new insights into pathogenesis and host defenses. Pathog. Dis. 67, 159-173. doi: 10.1111/ 2049-632x.12033 
Gonzalez Barrios, A. F., Zuo, R., Hashimoto, Y., Yang, L., Bentley, W. E., and Wood, T. K. (2006). Autoinducer 2 controls biofilm formation in Escherichia coli through a novel motility quorum-sensing regulator (MqsR, B3022). J. Bacteriol. 188, 305-316. doi: 10.1128/jb.188.1.305-316.2006

Hardie, K. R., and Heurlier, K. (2008). Establishing bacterial communities by 'word of mouth': LuxS and autoinducer 2 in biofilm development. Nat. Rev. Microbiol. 6, 635-643. doi: 10.1038/nrmicro1916

Hoffmann, N., Rasmussen, T. B., Jensen, P. O., Stub, C., Hentzer, M., Molin, S., et al. (2005). Novel mouse model of chronic Pseudomonas aeruginosa lung infection mimicking cystic fibrosis. Infect. Immun. 73, 2504-2514. doi: 10.1128/iai.73.4. 2504-2514.2005

Jang, Y. J., Choi, Y. J., Lee, S. H., Jun, H. K., and Choi, B. K. (2013). Autoinducer 2 of Fusobacterium nucleatum as a target molecule to inhibit biofilm formation of periodontopathogens. Arch. Oral. Biol. 58, 17-27. doi: 10.1016/j.archoralbio. 2012.04.016

Jiricny, N., Molin, S., Foster, K., Diggle, S. P., Scanlan, P. D., Ghoul, M., et al. (2014). Loss of social behaviours in populations of Pseudomonas aeruginosa infecting lungs of patients with cystic fibrosis. PLOS ONE 9:e83124. doi: 10.1371/journal. pone.0083124

Kong, K. F., Jayawardena, S. R., Indulkar, S. D., Del Puerto, A., Koh, C. L., Hoiby, N., et al. (2005). Pseudomonas aeruginosa AmpR is a global transcriptional factor that regulates expression of AmpC and PoxB betalactamases, proteases, quorum sensing, and other virulence factors. Antimicrob. Agents Chemother. 49, 4567-4575. doi: 10.1128/aac.49.11.4567-4575.2005

Li, H., Li, X., Wang, Z., Fu, Y., Ai, Q., Dong, Y., et al. (2015a). Autoinducer2 regulates Pseudomonas aeruginosa PAO1 biofilm formation and virulence production in a dose-dependent manner. BMC Microbiol. 15:192. doi: 10.1186/ s12866-015-0529-y

Li, H., Song, C., Liu, D., Ai, Q., and Yu, J. (2015b). Molecular analysis of biofilms on the surface of neonatal endotracheal tubes based on 16S rRNA PCR-DGGE and species-specific PCR. Int. J. Clin. Exp. Med. 8, 11075-11084.

Mathee, K., Narasimhan, G., Valdes, C., Qiu, X., Matewish, J. M., Koehrsen, M., et al. (2008). Dynamics of Pseudomonas aeruginosa genome evolution. Proc. Natl. Acad. Sci. U.S.A. 105, 3100-3105. doi: 10.1073/pnas.0711982105

McNab, R., Ford, S. K., El-Sabaeny, A., Barbieri, B., Cook, G. S., and Lamont, R. J. (2003). LuxS-based signaling in Streptococcus gordonii: autoinducer 2 controls carbohydrate metabolism and biofilm formation with Porphyromonas gingivalis. J. Bacteriol. 185, 274-284. doi: 10.1128/JB.185.1.274-284.2003

Miller, M. B., and Bassler, B. L. (2001). Quorum sensing in bacteria. Annu. Rev. Microbiol. 55, 165-199. doi: 10.1146/annurev.micro.55.1.165

Ochsner, U. A., and Reiser, J. (1995). Autoinducer-mediated regulation of rhamnolipid biosurfactant synthesis in Pseudomonas aeruginosa. Proc. Natl. Acad. Sci. U.S.A. 92, 6424-6428. doi: 10.1073/pnas.92.14.6424

Pan, Y., Du, L., Ai, Q., Song, S., Tang, X., Zhu, D., et al. (2017). Microbial investigations in throat swab and tracheal aspirate specimens are beneficial to predict the corresponding endotracheal tube biofilm flora among intubated neonates with ventilator-associated pneumonia. Exp. Ther. Med. 14, 1450-1458. doi: 10.3892/etm.2017.4631

Pearson, J. P., Gray, K. M., Passador, L., Tucker, K. D., Eberhard, A., Iglewski, B. H., et al. (1994). Structure of the autoinducer required for expression of
Pseudomonas aeruginosa virulence genes. Proc. Natl. Acad. Sci. U.S.A. 91, 197-201. doi: 10.1073/pnas.91.1.197

Roy, V., Meyer, M. T., Smith, J. A., Gamby, S., Sintim, H. O., Ghodssi, R., et al. (2013). AI-2 analogs and antibiotics: a synergistic approach to reduce bacterial biofilms. Appl. Microbiol. Biotechnol. 97, 2627-2638. doi: 10.1007/s00253-0124404-6

Sarkar, R., Chaudhary, S. K., Sharma, A., Yadav, K. K., Nema, N. K., Sekhoacha, M., et al. (2014). Anti-biofilm activity of Marula - a study with the standardized bark extract. J. Ethnopharmacol. 154, 170-175. doi: 10.1016/j.jep.2014. 03.067

Silby, M. W., Winstanley, C., Godfrey, S. A., Levy, S. B., and Jackson, R. W. (2011). Pseudomonas genomes: diverse and adaptable. FEMS Microbiol. Rev. 35, 652-680. doi: 10.1111/j.1574-6976.2011.00269.x

Singh, P. K., Parsek, M. R., Greenberg, E. P., and Welsh, M. J. (2002). A component of innate immunity prevents bacterial biofilm development. Nature 417, 552-555. doi: 10.1038/417552a

Song, Z., Zhang, J., Zhang, X., Li, D., Wang, H., Xu, X., et al. (2014). Interleukin 4 deficiency reverses development of secondary Pseudomonas aeruginosa pneumonia during sepsis-associated immunosuppression. J. Infect. Dis. 211, 1616-1627. doi: 10.1093/infdis/jiu668

Wang, Z., Xiang, Q., Yang, T., Li, L., Yang, J., Li, H., et al. (2016). Autoinducer-2 of Streptococcus mitis as a target molecule to inhibit pathogenic multi-species biofilm formation in vitro and in an endotracheal intubation rat model. Front. Microbiol. 7:88. doi: 10.3389/fmicb.2016.00088

Wu, H., Song, Z., Givskov, M., Doring, G., Worlitzsch, D., Mathee, K., et al. (2001). Pseudomonas aeruginosa mutations in lasI and rhlI quorum sensing systems result in milder chronic lung infection. Microbiology 147(Pt 5), 1105-1113. doi: 10.1099/00221287-147-5-1105

Yang, Y. X., Xu, Z. H., Zhang, Y. Q., Tian, J., Weng, L. X., and Wang, L. H. (2012). A new quorum-sensing inhibitor attenuates virulence and decreases antibiotic resistance in Pseudomonas aeruginosa. J. Microbiol. 50, 987-993. doi: 10.1007/s12275-012-2149-7

Yilmaz, Ö., Benedyk, M., Byrne, D. P., Glowczyk, I., Potempa, J., Olczak, M., et al. (2015). Pyocycanin, a contributory factor in haem acquisition and virulence enhancement of Porphyromonas gingivalis in the Lung. PLOS ONE 10:e0118319. doi: 10.1371/journal.pone.0118319

Yoshida, A., Ansai, T., Takehara, T., and Kuramitsu, H. K. (2005). LuxSbased signaling affects Streptococcus mutans biofilm formation. Appl. Environ. Microbiol. 71, 2372-2380. doi: 10.1128/aem.71.5.2372-2380.2005

Conflict of Interest Statement: The authors declare that the research was conducted in the absence of any commercial or financial relationships that could be construed as a potential conflict of interest.

Copyright (c) 2017 Li, Li, Song, Zhang, Wang, Liu, Wei and Yu. This is an open-access article distributed under the terms of the Creative Commons Attribution License (CC BY). The use, distribution or reproduction in other forums is permitted, provided the original author(s) or licensor are credited and that the original publication in this journal is cited, in accordance with accepted academic practice. No use, distribution or reproduction is permitted which does not comply with these terms. 